\title{
Differential effects of histone deacetylase inhibitors on cellular drug transporters and their implications for using epigenetic modifiers in combination chemotherapy
}

\author{
Benigno C. Valdez ${ }^{1}$, Yang Li ${ }^{1}$, David Murray ${ }^{2}$, Jonathan E. Brammer ${ }^{1}$, Yan Liu ${ }^{1}$, \\ Chitra Hosing ${ }^{1}$, Yago Nieto ${ }^{1}$, Richard E. Champlin ${ }^{1}$, Borje S. Andersson ${ }^{1}$ \\ ${ }^{1}$ Department of Stem Cell Transplantation and Cellular Therapy, University of Texas MD Anderson Cancer Center, Houston, \\ Texas 77030, USA \\ ${ }^{2}$ Department of Experimental Oncology, Cross Cancer Institute, Edmonton, Alberta T6G 1Z2, Canada \\ Correspondence to: Benigno C. Valdez, email: bvaldez@mdanderson.org \\ Keywords: HDAC inhibitors, drug transporter, combination therapy, lymphoma, pre-transplant regimen \\ Received: May 05, $2016 \quad$ Accepted: August 11, $2016 \quad$ Published: August 24, 2016
}

\section{ABSTRACT}

HDAC inhibitors, DNA alkylators and nucleoside analogs are effective components of combination chemotherapy. To determine a possible mechanism of their synergism, we analyzed the effects of HDAC inhibitors on the expression of drug transporters which export DNA alkylators. Exposure of PEER Iymphoma T-cells to $15 \mathrm{nM}$ romidepsin (Rom) resulted in 40\%-50\% reduction in mRNA for the drug transporter MRP1 and up to 500-fold increase in the MDR1 mRNA within 32-48 hrs. MRP1 protein levels concomitantly decreased while MDR1 increased. Other HDAC inhibitors panobinostat, belinostat and suberoylanilide hydroxamic acid (SAHA) - had similar effects on these transporters. The protein level of MRP1 correlated with cellular resistance to busulfan and chlorambucil, and Rom exposure sensitized cells to these DNA alkylators. The decrease in MRP1 correlated with decreased cellular drug export activity, and increased level of MDR1 correlated with increased export of daunorubicin. A similar decrease in the level of MRP1 protein, and increase in MDR1, were observed when mononuclear cells derived from patients with T-cell malignancies were exposed to Rom. Decreased MRP1 and increased MDR1 expressions were also observed in blood mononuclear cells from lymphoma patients who received SAHA-containing chemotherapy in a clinical trial. This inhibitory effect of HDAC inhibitors on the expression of MRP1 suggests that their synergism with DNA alkylating agents is partly due to decreased efflux of these alkylators. Our results further imply the possibility of antagonistic effects when HDAC inhibitors are combined with anthracyclines and other MDR1 drug ligands in chemotherapy.

\section{INTRODUCTION}

Most chemotherapy drugs with different mechanisms of action are more effective when used in combination. Their combined cytotoxicity efficiently kills tumor cells, decreases the possibility of developing resistant cells, and provides patient safety when given at optimal doses. However, combined drugs may also have antagonistic effects, and this possibility underscores the importance of understanding their interactions.

Combination chemotherapy is a common part of pre-transplant conditioning regimens for patients undergoing hematopoietic stem cell transplantation
(HSCT). DNA alkylating agents, nucleoside analogs, epigenetic modifiers, and proteasome inhibitors, among others, have been combined to minimize cancer burden prior to HSCT [1]. The synergistic effects of some of these drugs involve alteration of the chromatin structure, activation of the DNA-damage response, inhibition of DNA repair, disruption of mitochondrial membrane, and activation of apoptosis $[1,2]$. Pre-clinical and clinical studies by our group showed the synergistic cytotoxicity of combined DNA alkylating agents busulfan $(\mathrm{Bu})$ and melphalan (Mel), nucleoside analog gemcitabine (Gem), and an HDAC inhibitor in lymphoma cells [3-5]. We proposed a mechanism of their synergism that includes 
Gem-mediated inhibition of DNA synthesis resulting in histone modifications and opening of chromatin structure which allows the genomic DNA to be more accessible to the alkylating agents $\mathrm{Bu}$ and $\mathrm{Mel}[1,2]$. These alkylating drugs exacerbate DNA damage and activate apoptosis. Addition of an HDAC inhibitor (eg. suberoylanilide hydroxamic acid, SAHA) facilitates histone modifications and chromatin remodeling, in addition to possible increased expression of tumor suppressor genes, and further enhances $\mathrm{Bu}$ and $\mathrm{Mel}$ adduct formation.

In an effort to identify additional mechanisms of their synergism, we hypothesized that HDAC inhibitors may affect the expression of drug transporters, which are responsible for the efflux of structurally diverse anti-cancer drugs including DNA alkylators. The best characterized are the MRP1/ABCC1, MDR1/P-glycoprotein/ABCB, and BCRP/ABCG2; their expressions may correlate with multidrug resistance [6]. Although there is a significant overlap in their substrate specificity, MRP1 exports a broader range of therapeutic drugs including their glutathione (GSH)-conjugates [7-9].

Our present study shows that HDAC inhibitors down-regulate expression of the $M R P 1$ gene and upregulate the MDR1 gene. Since MRP1 exports GSHconjugated DNA alkylators [7], a decrease in its protein level may contribute to the synergism of HDAC inhibitors and DNA alkylating agents. Conversely, HDAC inhibitors might antagonize the efficacy of anti-cancer drugs that are substrates for MDR1. These differential effects of HDAC inhibitors on the expression of drug transporters underscore the necessity for caution in combining these drugs with other chemotherapeutic agents.

\section{RESULTS}

\section{HDAC inhibitors decrease the expression of $M R P 1$ but increase $M D R 1$ expression}

The HDAC inhibitor Romidepsin (Rom) has been reported to increase the expression of $M D R 1$ in patient mononuclear cells [10], but whether and how this drug affects the expression of other drug transporters is unknown. We, therefore, examined the effects of Rom and panobinostat (Pano) on the expression of three drug transporter genes - MRP1, MDRl and $B C R P$ - at various time points in the PEER lymphoma cell line. Figure 1a shows similar effects of these two HDAC inhibitors; MRP1 protein levels started to decrease after 24-hr drug exposure and were almost eliminated after $48 \mathrm{hrs,} \mathrm{while}$ MDR1 protein levels started to increase after 32-hr drug exposure. On the other hand, BCRP protein levels slightly decreased after 48 hrs. Acetylation of histone 3 at Lys 9 (AcH3K9) started to increase after $24 \mathrm{hrs,} \mathrm{suggesting}$ the efficacy of Rom and Pano in inhibiting histone deacetylation. To determine if the effects of Rom and Pano on the expression of MRP1 and MDR1 were manifested at the transcription level, quantitative real-time PCR was performed. Figure $1 \mathrm{~b}$ shows $\sim 40 \%$ and $\sim 50 \%$ decrease in the mRNA level of MRP1 after 24- and 32-hr Rom exposure, respectively; some recovery was apparent after 48 hrs. Maximum effect of Pano on the MRP1 mRNA was observed after $24 \mathrm{hrs}$ and transcript levels started to recover after $32 \mathrm{hrs}$ (Figure 1b). The mRNA level of MDR1 continued to increase from 24 to $48 \mathrm{hrs}$ in the presence of either drug (Figure 1c).

SAHA, an HDAC inhibitor, is a commonly used anti-neoplastic agent [11]. We, therefore, sought to determine if SAHA and belinostat (Bel) would have similar effects on the expression of MRP1 and MDRl as Rom and Pano. We used drug concentrations approximately equivalent to their $\mathrm{IC}_{50}$ in the MTT assay (Figure 2a). At these concentrations apoptosis was activated as suggested by $\sim 60 \%$ Annexin $\mathrm{V}$ positivity (Figure $2 \mathrm{a}$ ) and cleavage of PARP1 and caspase 3 (Figure 2b). Again, MRP1 protein levels decreased in cells exposed to these HDAC inhibitors; MDR1 increased except in cells exposed to Bel (Figure 2b). DNA-damage response was activated as shown by increased phosphorylation of H2AX (Figure 2b). All four drugs inhibited histone deacetylase activity as suggested by increased levels of AcH3K9 with a corresponding increase in the methylation of histone 3 (Figure 2b). Additional Western blot analysis suggests (a)

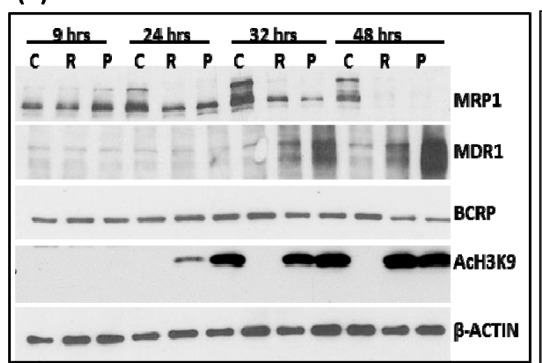

(b)

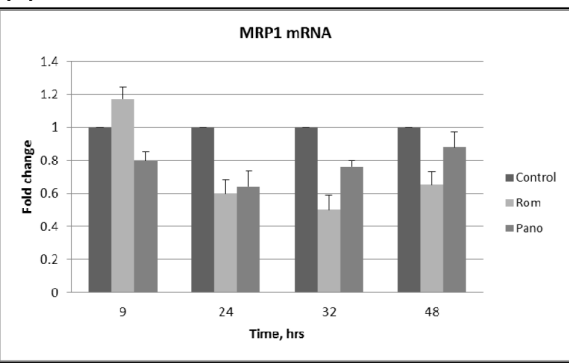

(c)

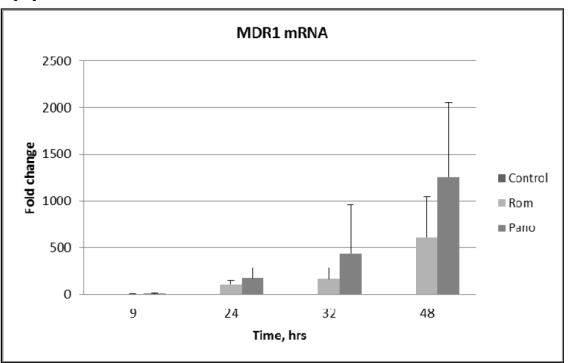

Figure 1: Kinetics of expression of MRP1, MDR1 and BCRP. PEER cells were exposed to solvent (C, control), $15 \mathrm{nM} \mathrm{romidepsin}$ (R, Rom) or $150 \mathrm{nM}$ panobinostat (P, Pano) and harvested after the indicated time (hrs). Total proteins and RNA were isolated and analyzed by Western blotting $\mathbf{a}$. and quantitative real time-PCR $\mathbf{b}$ and $\mathbf{c}$. respectively. 
that the observed increase in the level of AcH3K9 might be due to a decrease in the level of Class I and Class II histone deacetylases (Figure 2c). The phosphorylation of HDACs 3, 4, 5 and 7, which decreased in the presence of Rom, Bel, Pano and SAHA may also regulate the expression of MRP1 and MDR 1 genes. Our data suggest that these HDAC inhibitors decrease expression of Class I and II HDAC with a concomitant increase in acetylated histone 3 and a corresponding down-regulation of MRP1 but up-regulation of $M D R 1$.
The observed down-regulation of HDAC4, -5, -6 and -7 , which all belong to Class II, prompted us to determine if Class II selective inhibitors would similarly inhibit the expression of $M R P 1$ and up-regulate the expression of MDR1. Western blot analysis shows a decrease in the level of MRP1 protein in cells exposed to $5 \mu \mathrm{M}$ LMK-235, a selective inhibitor of HDAC4 and -5, but not in cells exposed to $0.5-1 \mu \mathrm{M}$ LMK-235 nor 5-10 $\mu \mathrm{M}$ Nexturastat A (NA), a selective inhibitor of HDAC6; the level of MDR1 protein was not significantly affected (a)

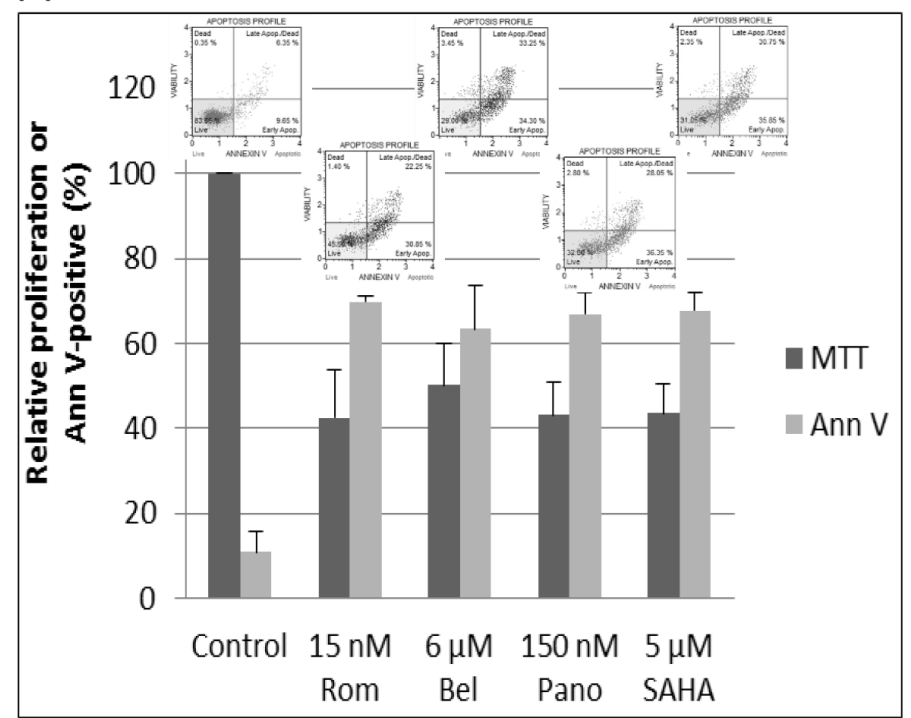

(c)

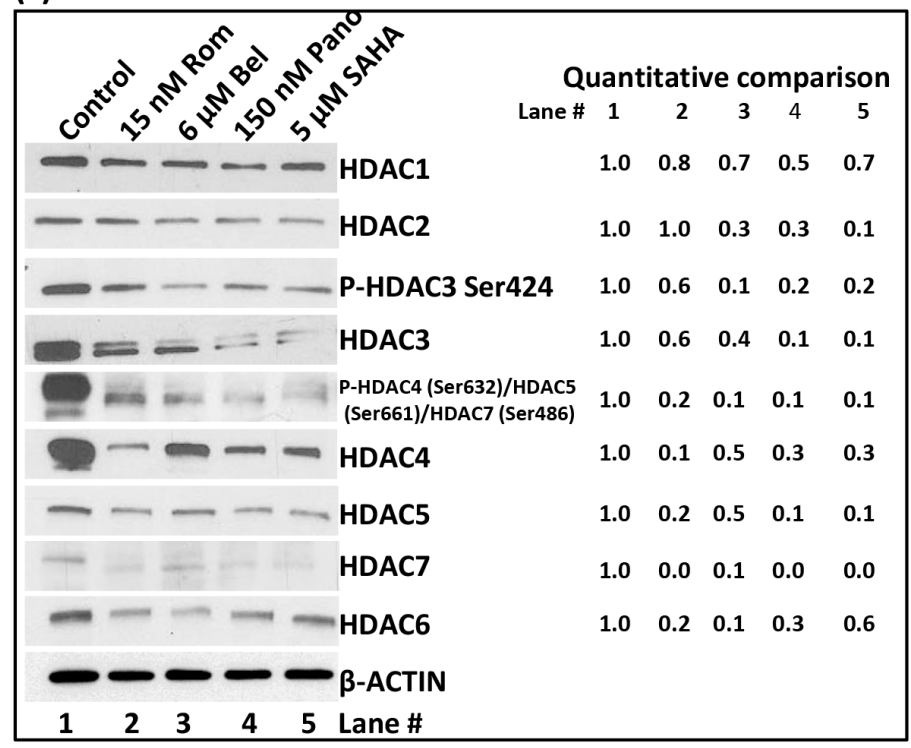

(b)

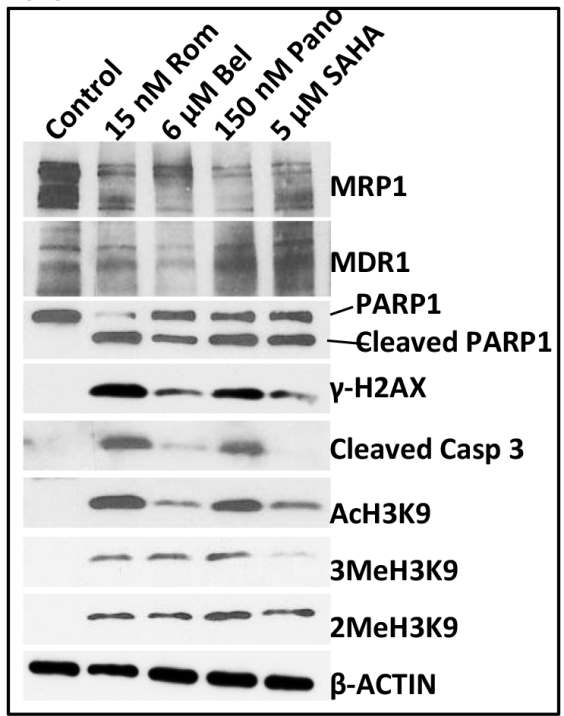

(d)

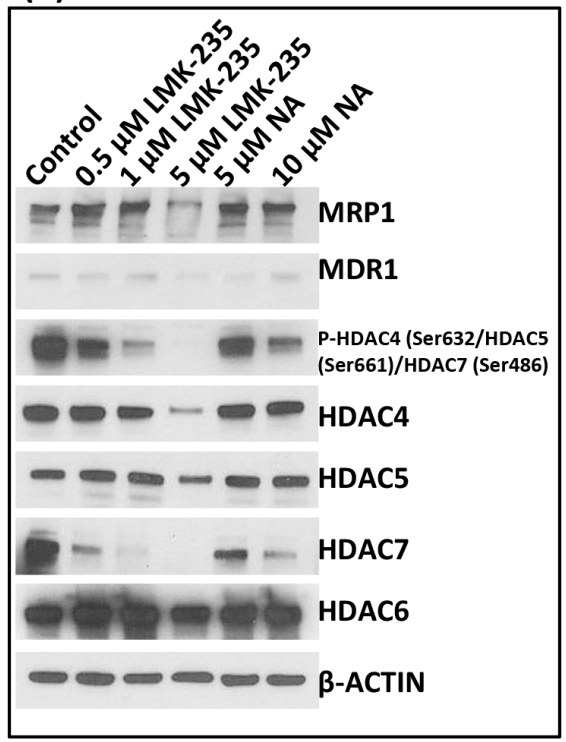

Figure 2: Effects of various histone deacetylase inhibitors on cell proliferation, apoptosis activation and histone modifications. PEER cells were exposed to drugs for $48 \mathrm{hrs}$ and analyzed for proliferation using the MTT assay a. Activation of apoptosis was determined using the Annexin V (Ann V; flow cytometry dot plots showing apoptosis profile for each treatment are on top of the bar graphs) assay (a) and Western blotting b. Modifications of histone 3 (b) and changes in the level of different histone deacetylases c. were determined by Western blotting. Quantitative analysis of the bands was done by normalizing the signals to $\beta$-ACTIN and calculating the ratio relative to the control (left side of panel c). d. Cells were exposed to HDAC Class II selective inhibitors LMK-235 or Nexturastat A (NA) at the indicated concentrations for 48 hrs and analyzed by Western blotting. Rom, romidepsin; Bel, belinostat, Pano, panobinostat; SAHA, suberoylanilide hydroxamic acid. 
(Figure 2d). The phosphorylation status of HDAC4, 5 and 7 dramatically decreased in cells exposed to $0.5-5 \mu \mathrm{M}$ LMK-235 and 5-10 $\mu \mathrm{M}$ NA but their respective level of total protein significantly changed only at $5 \mu \mathrm{M}$ LMK-235 (Figure 2d). The level of HDAC6 was not significantly affected by either drug. These results suggest that among the members of Class II group HDAC4, -5 and -7 but not HDAC6 may be involved in the regulation of the MRP1 gene expression but not MDRl.

\section{HDAC inhibitors decrease MRP1 activity}

At the molecular level, RT-PCR and Western blot analyses showed decreased levels of MRP1 mRNA and protein. We next sought to determine if cells exposed to HDAC inhibitors would have a decreased functional ability to export specific drugs using flow cytometry. We initially analyzed the kinetics of efflux of CFDA, a known substrate for MRP1 [12], in PEER cells previously exposed to solvent or $15 \mathrm{nM}$ Rom as described in Figure 3. Cells were kept on ice and allowed to take up CFDA for $20 \mathrm{~min}$, then shifted to $37^{\circ} \mathrm{C}$ to allow efflux of the fluorescent substrate for various periods of time. The control cells exported $\sim 33 \%-73 \%$ of CFDA after 15 $25 \mathrm{~min}$, increasing to $\sim 92 \%$ efflux after $30 \mathrm{~min}$ (Figure $3 a)$. On the other hand, cells previously exposed to Rom showed only $\sim 47 \%$ efflux of CFDA after 30 min (Figure $3 \mathrm{a}$ ), suggesting almost $50 \%$ inhibition of the CFDA efflux in Rom-treated cells. To confirm if efflux of CFDA was mediated by MRP1, cells were exposed to MK571, a known inhibitor of MRP1 [13]; efflux was indeed inhibited by $\sim 40 \%$ compared to control cells (Figure $3 \mathrm{~b}$ ).
Rom (15 nM) and $100 \mathrm{nM}$ Pano decreased CFDA efflux from $\sim 97 \%$ (Control) to $\sim 55 \%$ and $\sim 74 \%$, respectively. Again, the drug-mediated decrease in MRP1 protein level was confirmed by Western blotting (Figure 3c). It is not clear why exposing PEER cells to $100 \mathrm{nM}$ Pano almost eliminated MRP1 protein (Figure 3c) but inhibited CFDA efflux by only $\sim 23 \%$ (Figure $3 \mathrm{~b}$ ). Overall, these results show strong correlation of decreased MRP1 mRNA/ protein levels and transport activity in cells exposed to HDAC inhibitors.

\section{HDAC inhibitors increase MDR1 activity}

We similarly analyzed the effects of HDAC inhibitors on the drug transport activity of MDR1 in U937 cells by flow cytometry using $\operatorname{DiOC}_{2}(3)$, a known substrate for MDR1 [14]. Exposure of cells to $7 \mathrm{nM}$ Rom or $45 \mathrm{nM}$ Pano decreased MRP1 but increased MDR1 protein levels (Figure 4a, inset). Efflux of $\mathrm{DiOC}_{2}(3)$ from cells kept on ice during the efflux assay was not significant for either control or drug-treated cells; shifting to $37^{\circ} \mathrm{C}$ showed almost negligible efflux of $\mathrm{DiOC}_{2}(3)$ from the control cells but efflux increased to $\sim 39 \%$ and $\sim 30 \%$ for cells pre-exposed to Rom and Pano, respectively (Figure 4a). The observed efflux activity correlated with the level of MDR1 protein as shown by Western blotting.

Since MDR1 is known to catalyze export of anthracyclines such as the anti-neoplastic drugs daunorubicin and doxorubicin [15], we determined if daunorubicin efflux would be increased in U937 cells pre-exposed to Rom or Pano. The decrease in cellular daunorubicin fluorescence, as measured by flow cytometry, (a) Kinetics of CFDA Efflux

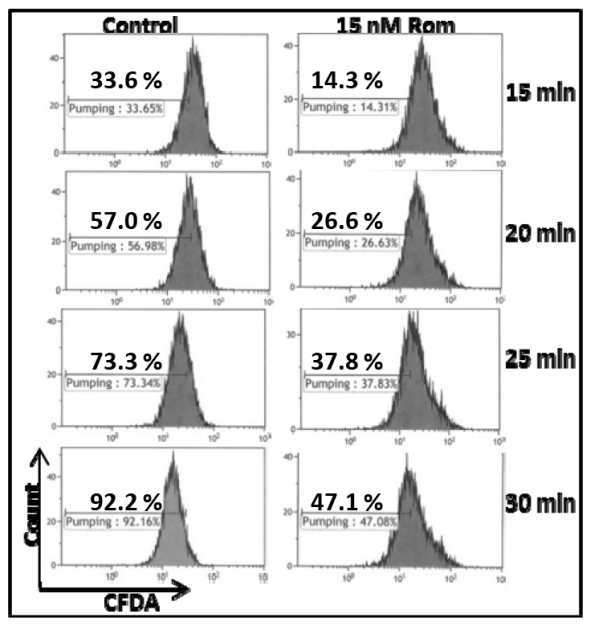

(b) Drug-mediated inhibition of CFDA efflux

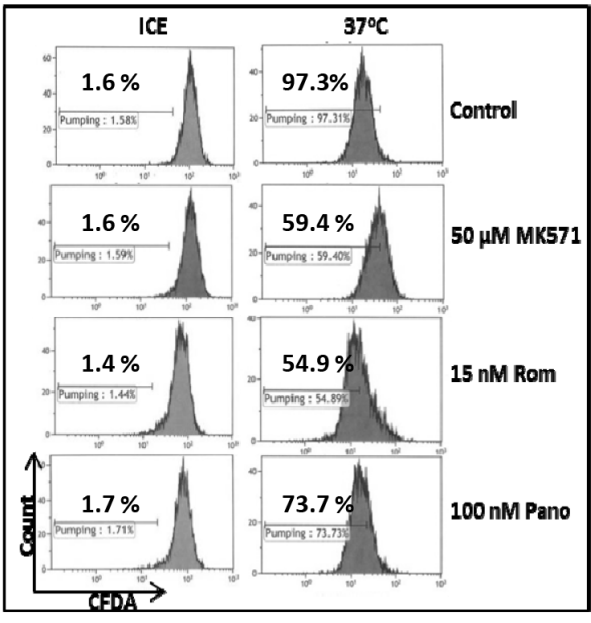

(c) Western blot

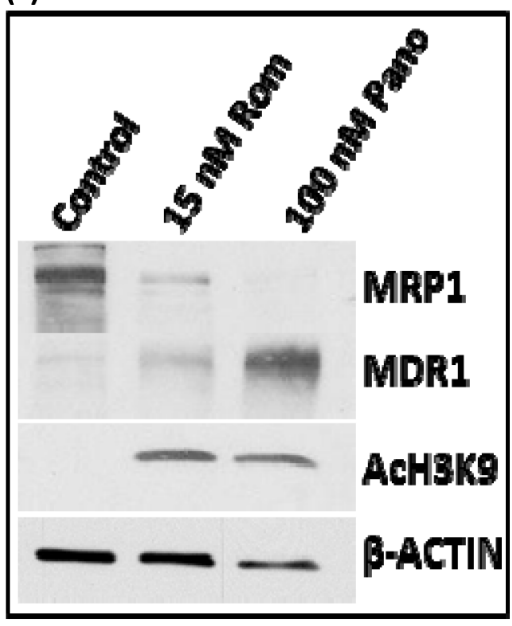

Figure 3: Functional assay of MRP1 protein. PEER cells were exposed to solvent (Control) or $15 \mathrm{nM}$ romidepsin (Rom) for $48 \mathrm{hrs}$, harvested, incubated with the MRP1 substrate CFDA for $20 \mathrm{~min}$, shifted to $37^{\circ} \mathrm{C}$, and analyzed for efflux of CFDA at the indicated time point by flow cytometry a. To determine drug-mediated inhibition of CFDA efflux, cells were exposed to $15 \mathrm{nM}$ Rom or $100 \mathrm{nM}$ Pano (panobinostat) for $48 \mathrm{hrs}$ or to the MRP1 inhibitor MK571 (50 $\mathrm{MM})$ for $30 \mathrm{~min}$ prior to the efflux assay. CFDA efflux was performed for 30 min b. "Count" refers to the number of live (i.e., PI-negative) cells. Western blot analysis confirmed the 48-hr drug-mediated decrease in MRP1 protein level c. 
was used as an indicator of drug efflux. Cells were allowed to take up daunorubicin for $1.5 \mathrm{hrs}$ at $37^{\circ} \mathrm{C}$, washed and incubated in drug-free culture medium. Approximately $30 \%$ efflux of daunorubicin from the control cells occurred after 3 hrs. Daunorubicin efflux increased to $\sim 58 \%$ and $\sim 55 \%$ in cells pre-exposed to Rom and Pano, respectively (Figure 4b). Addition of orthovanadate, a known inhibitor of ATPase-dependent drug transporters [16, 17], abrogated the Rom- and Pano-mediated increase in efflux of daunorubicin (Figure 4b). Overall, these results show that HDAC inhibitors increase the drug transport activity of MDR1, consistent with increased MDR1 mRNA and protein levels (Figure 1).

\section{The level of expression of $M R P 1$ correlates with cellular sensitivity to DNA alkylating agents}

DNA alkylating agents such as $\mathrm{Bu}$ and Mel exert synergistic cytotoxicity when combined with HDAC inhibitors in pre-clinical and clinical studies [3-5]. Moreover, GSH-conjugated alkylators are known to be exported from cells via the MRP1 drug transporter [7]. Based on these previous studies and on the observed downregulation of $M R P 1$ at the mRNA, protein and functional levels, we hypothesized that treatment of cells with HDAC inhibitors may result in inhibition of the efflux of GSHconjugated alkylators. To test this hypothesis, we first examined the correlation between the basal level of MRP1 and the cellular sensitivity to DNA alkylating agents of PEER (T-cell leukemia), Daudi (B-cell lymphoma) and MV4-11 (AML) cell lines. The level of expression of MRP1 was highest in PEER and lowest in MV4-11 cells (Figure 5a). Dose-response analysis showed highest resistance of PEER cells to two DNA alkylating agents, busulfan and chlorambucil, and least resistance of MV411 cells (Figure 5b and 5c). PEER cells became more sensitive to chlorambucil (Figure 5d) and busulfan (data not shown) in the presence of Rom. Whereas proliferation of PEER cells was not significantly affected by $0-10 \mu \mathrm{M}$ chlorambucil (slope of the dose-response line $=-0.05$ ), a steeper slope of -1.6 was obtained in the presence of $7 \mathrm{nM}$ Rom, suggesting a Rom-mediated decrease in resistance of PEER cells to chlorambucil (Figure 5d). Again, exposure of all three cell lines to Rom or Pano decreased the level of MRP1 protein (Figure 5e). The observed correlation between DNA alkylator sensitivity and the level of MRP1 protein is consistent with the previous report that MRP1 exports DNA alkylators from cells [7, 8], and suggests that the synergism of these drugs with HDAC inhibitors is partly due to down-regulation of the expression of MRP1.

\section{HDAC inhibitors down-regulate $M R P 1$ and up- regulate $M D R 1$ in patient-derived cell samples}

To determine the potential clinical significance of our cell line studies, we exposed mononuclear cells isolated from the peripheral blood of patients with T-cell malignancies (patient characteristics are shown in Table 1, Supplementary Materials) to Rom and analyzed them by Western blotting. The MRP1 protein levels decreased in a Rom concentration-dependent manner while the MDR1 protein levels increased in both patient samples (Figure $6 a)$. While the level of BCRP did not change in sample L-02, it significantly decreased in sample B-03 in the presence of Rom (Figure 6a). We extended this study to an analysis of mononuclear cells from patients enrolled in a clinical trial evaluating SAHA, azacytidine, Gem, Bu and $\mathrm{Mel}$ as a pre-transplant conditioning regimen [18]. Patients (a)

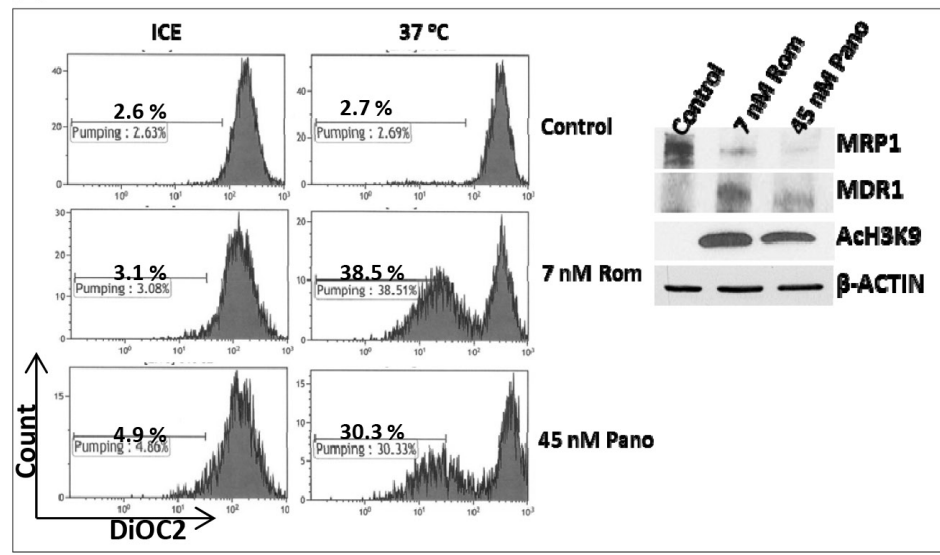

(b)

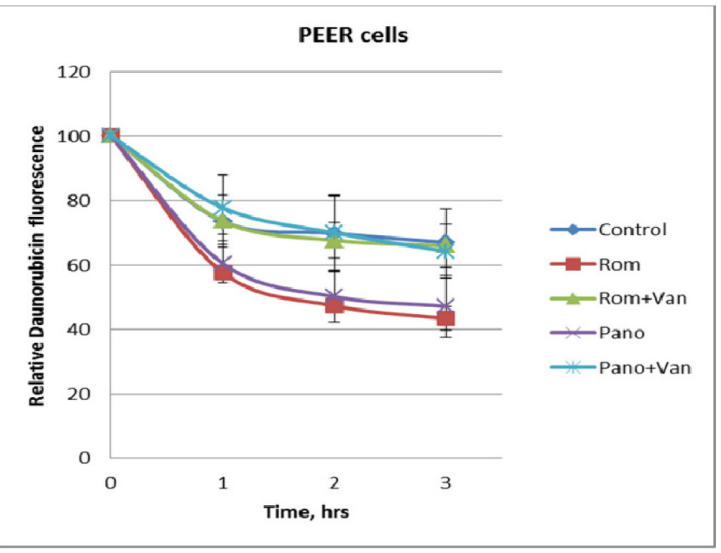

Figure 4: Functional assay of MDR1 protein. a. U937 cells were exposed to solvent (Control), $7 \mathrm{nM}$ romidepsin (Rom) or $45 \mathrm{nM}$ panobinostat (Pano) for $48 \mathrm{hrs}$, harvested, incubated with MDR1 substrate $\mathrm{DiOC}_{2}(3)$ for $20 \mathrm{~min}$, shifted to $37^{\circ} \mathrm{C}$ for 45 min and analyzed for efflux of $\operatorname{DiOC}_{2}(3)$ by flow cytometry as described under Materials and Methods. "Count" refers to the number of live (i.e., DAPInegative) cells. Total protein extracts were analyzed by Western blotting (inset) to determine changes in the level of MDR1 protein. b. A similar analysis was performed using daunorubicin as a fluorescent substrate for MDR1 in the absence or presence of $2 \mathrm{mM}$ orthovanadate (Van), an MDR1 inhibitor. 
(a)

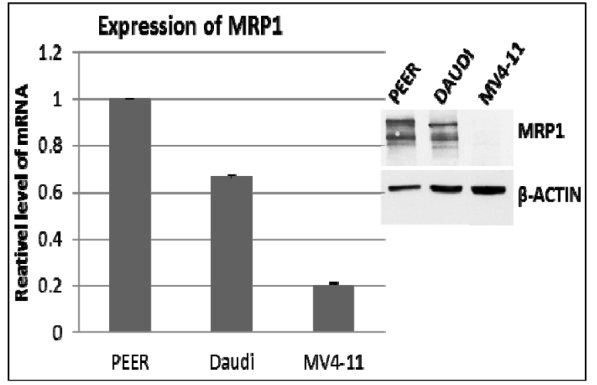

(d)

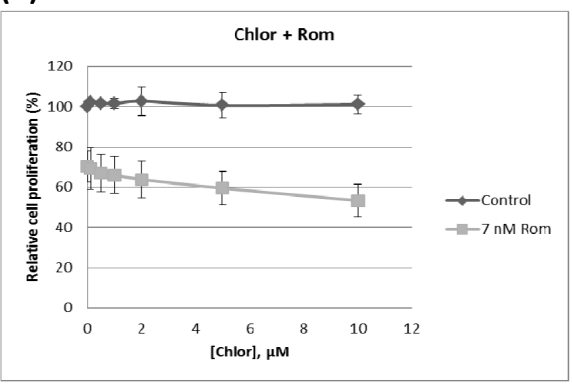

(b)

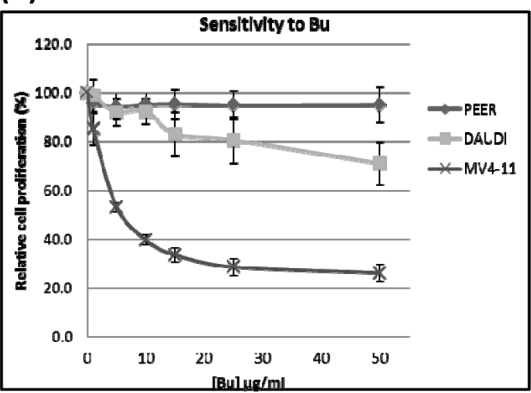

(e) (c)

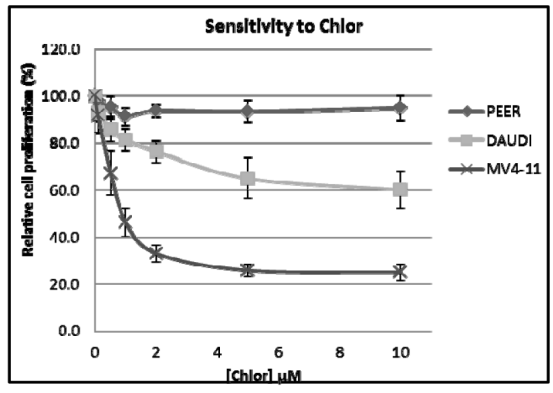

Figure 5: Correlation between the level of MRP1 protein and cell sensitivity to DNA alkylating agents. a. The expression of MRP1 in PEER, Daudi and MV4-11 cells was determined by quantitative real time-PCR (mRNA level) and Western blotting (protein level, inset). b, c. The three cell lines were exposed to various concentrations of busulfan (Bu) or chlorambucil (Chlor) for 48 hrs and analyzed for cell proliferation using the MTT assay. d. PEER cells were exposed to Chlor with or without $7 \mathrm{nM}$ Rom for 48 hrs and analyzed by the MTT assay. e. Cells were exposed to HDAC inhibitors (inset: drug concentrations) for 48 hrs and total protein extracts were analyzed by Western blotting.

(a) In vitro drug exposure of patient-derived cell samples

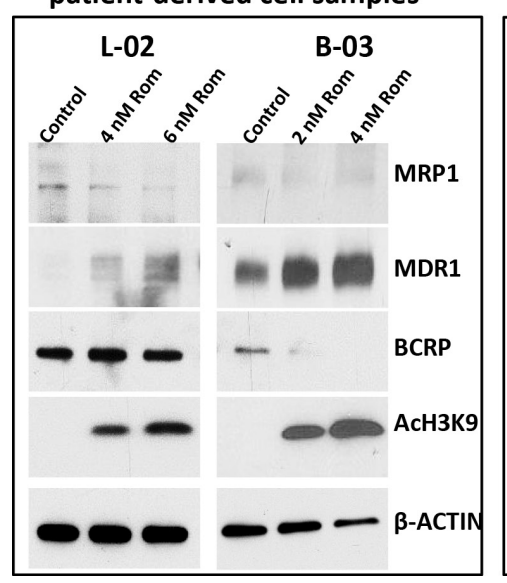

(b) Mononuclear cells from patients enrolled in clinical protocol

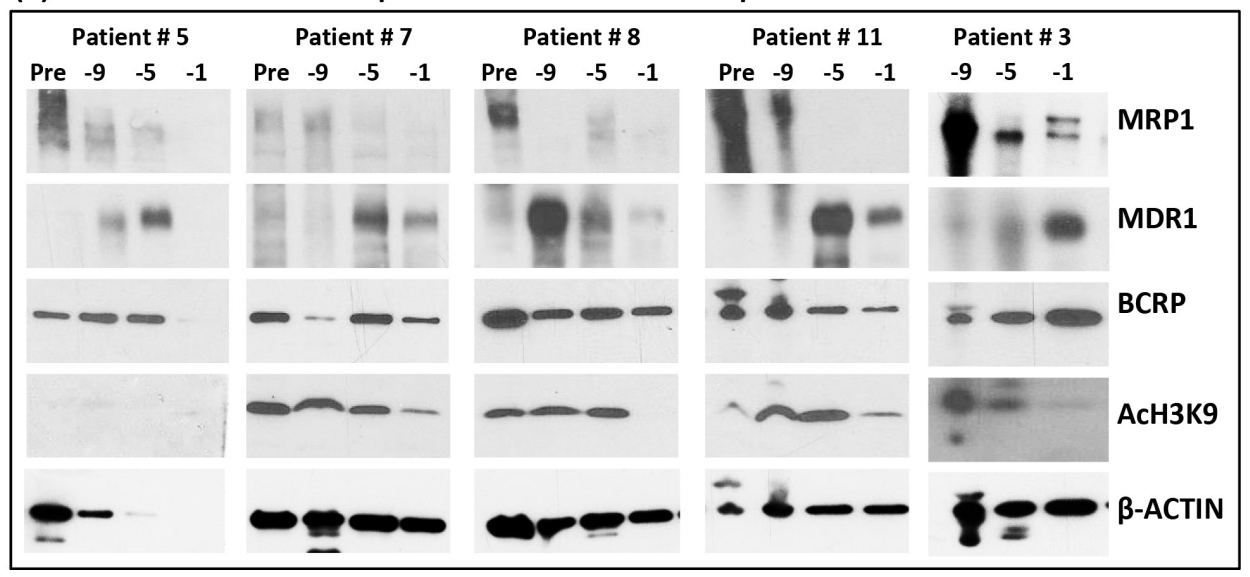

Figure 6: Effects of HDAC inhibitors on patient-derived cell samples. a. Mononuclear cells from blood of patients with peripheral T-cell lymphoma (L-02) and T-cell prolymphocytic leukemia (B-03) were isolated and exposed to the indicated concentrations of romidepsin (Rom) for $48 \mathrm{hrs}$, and total protein extracts were analyzed by Western blotting. b. Mononuclear cells from patients enrolled in clinical trial NCT01983969 were isolated and analyzed by Western blotting. These patients received a pretransplant conditioning regimen that included gemcitabine, busulfan, melphalan, azacytidine and SAHA. SAHA (Vorinostat) was given on days -11 to -3 prior to hematopoietic stem cell transplantation. Baseline samples (Pre) were taken before the conditioning therapy and treated samples at 9, 5,1 day(s) prior to transplant. Sampling prior to chemotherapy for patient \#3 was not performed for logistical reasons. 
received SAHA starting at day -11 prior to stem cell transplantation. Figure $6 \mathrm{~b}$ shows a decrease in the MRP1 protein level from day -9 to day -1 relative to its baseline level (pre-chemotherapy) while the MDR1 protein level increased in all 5 patient samples and the BCRP level was not greatly affected. These results are consistent with our in vitro cell line and patient cell sample studies (Figures 1 and $6 a)$, suggesting that HDAC inhibitors differentially affect the expression of MRP1 and MDR1 both in in vitro and in vivo settings.

\section{DISCUSSION}

We previously demonstrated the synergistic cytotoxicity of DNA alkylating agents, nucleoside analogs and HDAC inhibitors in various experimental models [3-5]. Here, we present a novel mechanism of their synergism in established cell lines and patient-derived cell samples. HDAC inhibitors were seen to down-regulate the expression of $M R P 1$, resulting in decreased MRP1 protein levels and drug transport activity (Figures 1, 3). Since MRP1 is known to pump GSH-conjugated DNA alkylators out of cells [7], decreased MRP1 activity may cause cellular accumulation of DNA alkylating agents and thus enhanced cytotoxicity. This inference is consistent with (1) a strong inverse correlation between the level of MRP1 protein and sensitivity to alkylating agents; cells with high levels of MRP1 are more resistant to Bu and chlorambucil, but this phenotype is reversed when cells are exposed to Rom (Figure 5), and (2) feedback inhibition of glutathione S-transferases by the GSH-DNA alkylator conjugates, leading to increased intracellular concentrations of free alkylating agents [19].

The inhibitory effect of HDAC inhibitors on MRP1 expression is apparent at the transcription level (Figure 1b). Post-transcriptional regulation(s), however, may also contribute to these effects, as suggested by a greater decrease in the MRP1 protein compared with its mRNA levels (Figure 1). Changes in the acetylation status of proteins involved in the translation of MRP1 mRNA, and possibly MRP1 protein itself, may contribute to the reduction of MRP1 levels. Rom and Pano down-regulate $M R P 1$ expression within $24 \mathrm{hrs}$ of drug exposure as indicated by decreased mRNA levels. These changes coincide with histone modifications (Figure 1a), which may inhibit or enhance transcription $[20,21]$. Acetylation and methylation of histone 3 are both enhanced in PEER cells exposed to Rom, Bel, Pano and SAHA (Figure 2b); acetylation is partly due to decreased protein levels of HDACs 3, 4 and 6 (Figure 2c), and methylation is most likely due to the known functional interaction between histone methyltransferases and deacetylases [22]. It remains to be determined which histone modifications contribute to the inhibition of $M R P 1$ expression.

Similar results were observed in patient-derived cell samples. Exposure of cells, obtained from patients with peripheral T-cell lymphoma and T-cell prolymphocytic leukemia, to Rom decreased the MRP1 protein level (Figure 6a). Mononuclear cells isolated from patients treated with a SAHA-containing chemotherapy regimen also showed decreased MRP1 relative to samples taken prior to chemotherapy (Figure 6b). These data show that both in vitro and in vivo exposures of cells to HDAC inhibitors resulted in down-regulation of the MRP1 gene.

While HDAC inhibitors down-regulate the MRP1 gene, they up-regulate the expression of the MDR1 gene. This effect is also apparent at the transcription level within $24 \mathrm{hrs}$ of drug exposure (Figure 1c) with a concomitant increase in protein levels and drug transport activity of MDR1 (Figure 1a and 4c). Similar results were obtained in established cell lines and patient-derived cell samples, suggesting general differential effects of HDAC inhibitors on the expression of drug transporter genes. The mechanisms underlying these differential effects remain unknown, but may be related to differences in the sitespecific acetylation/methylation of histones. For example, acetylation and monomethylation of histone 3 at Lys 9 activate gene transcription whereas di- and tri-methylation of the same residue results in repression [23-25]. Changes in histone modifications may also affect expression of activators and repressors of gene transcription, as well as mediating a switch between transcription permissive and repressive chromatin structures [20].

Our study provides a potentially novel approach to reverse drug resistance in patients. While few inhibitors which directly bind to drug transporters such as verapamil, phenothiazine and cyclosporine D derivatives [26] have been unsuccessful in overcoming drug resistance in the clinic albeit their efficacy in laboratory models, the use of HDAC inhibitors to down-regulate MRP1 expression occurs at the gene level. In fact, our clinical study shows SAHA-mediated down-regulation of MRP1 expression (Figure 6b). Although a well-designed and controlled clinical trial is needed to unequivocally prove the role of HDAC inhibitors in the reversal of MRP1-mediated drug resistance in the clinic, our laboratory and clinical data suggest its possibility.

In summary, our results present potentially critical implications for combining HDAC inhibitors with other chemotherapy agents. Combination of HDAC inhibitors with DNA alkylators and other MRP1 substrates is expected to result in synergistic and more efficacious outcomes. However, the increased MDR1 expression induced by HDACi may result in reduced anticancer activity of MDR1 substrates, such as anthracyclines, vinca alkaloids and steroids. Our data seem timely and relevant in light of a currently ongoing clinical trial (NCT01280526), testing the value of combined romidepsin and CHOP (cyclophosphamide, adriamycin, vincristine, and prednisone). In particular, efflux of adriamycin, vincristine and prednisone may increase with HDAC inhibitor-mediated up-regulation of the MDR1 
gene [6]. Various drug combinations are used in pretransplant conditioning prior to hematopoietic stem cell transplantation, and our study highlights the importance of understanding the mechanism(s) of drug interactions to achieve a more efficacious cytotoxicity to tumor cells.

\section{MATERIALS AND METHODS}

\section{Cells and drugs}

PEER is a cell line originally isolated and established from a patient with T-cell acute lymphoblastic leukemia [27], Daudi (ATCC, Manassas, VA) is a B-lymphoblast cell line, U937 (ATCC) is a cell line originally established from a patient with histiocytic lymphoma [28], and MV411 (ATCC) is a biphenotypic B myelomonocytic leukemia cell line. Cells were grown in RPMI 1640 medium (Mediatech, Manassas, VA) supplemented with 10\% heatinactivated fetal bovine serum (Sigma-Aldrich, St Louis, $\mathrm{MO}$ ) and $100 \mathrm{U} / \mathrm{ml}$ penicillin and $100 \mu \mathrm{g} / \mathrm{ml}$ streptomycin (Mediatech) at $37^{\circ} \mathrm{C}$ in a fully humidified atmosphere of $5 \% \mathrm{CO}_{2}$ in air. Romidepsin (Rom), panobinostat (Pano), belinostat (Bel), SAHA, LMK-235 and Nexturastat A were purchased from Selleck Chemicals (Houston, TX) and dissolved in dimethyl sulfoxide (DMSO) to prepare stock solutions.

\section{Patient samples}

Two lymphocytic leukemia cell samples (L-02 and B-03) were isolated from patient peripheral blood using lymphocyte separation medium (Mediatech) and incubated in suspension in the same RPMI 1640 medium described above. Patient L-02 had leukemic peripheral T-cell lymphoma, whereas patient B-03 had T-cell prolymphocytic leukemia in the setting of Li-Fraummeni syndrome (congenital p53 deficiency). Cells were exposed to DMSO or DMSO/romidepsin for $48 \mathrm{hrs}$ and analyzed by Western blotting.

Mononuclear cells from peripheral blood of patients enrolled in clinical trial NCT01983969 (PI: Dr. Yago Nieto) were isolated using lymphocyte separation medium (Mediatech), lysed and analyzed by Western blotting.

All patient samples were collected after obtaining written informed consent, and all studies using these patient samples were performed under a protocol approved by the Institutional Review Board of the University of Texas MD Anderson Cancer Center, in accordance with the Declaration of Helsinki.

\section{Western blot analysis}

Cells were exposed continuously to drugs for 48 hrs, harvested, washed with cold phosphate-buffered saline (PBS), and lysed with lysis buffer (Cell Signaling Technology, Danvers, MA). Cell extracts were analyzed for protein concentrations using a BCA Protein Assay kit (ThermoFisher Scientific, Rockford, IL), and immunostaining by Western blot was done as previously described [28]. The antibodies used in this study are listed in Table 2 (Supplementary Materials).

\section{Quantitative real-time PCR analysis}

Total RNA was purified using the RNeasy mini kit (Qiagen, Valencia, CA) and complementary DNA was synthesized using the High Capacity cDNA Reverse Transcription Kit with RNase Inhibitor (Applied Biosystems, Foster City, CA). Gene expression was determined by RT-PCR analysis using the iTAQ Universal SYBR Green Supermix (Bio-Rad Lab, Hercules, CA) as described [29]. The primers used are listed in Table 3 (Supplementary Materials).

\section{Cell proliferation and cell death assays}

Cell proliferation assays were done in triplicate using 96-well plates. Cells $\left(4 \times 10^{4}\right)$ in $100 \mu 1$ medium were incubated with or without the test HDACinhibiting drug at $37^{\circ} \mathrm{C}, 5 \% \mathrm{CO}_{2}$ for $48 \mathrm{hrs}$ and analyzed for proliferation using the 3-(4, 5-dimethylthiazol-2yl)-2, 5-diphenyl tetrazolium bromide (MTT) assay as previously described [29]. Programmed cell death was determined using the Annexin V assay which measures the externalization of phosphatidylserine by flow cytometry as described [30].

\section{Functional assays for MRP1 and MDR1}

5-Carboxyfluorescein diacetate (CFDA from Sigma-Aldrich) and 3, 3'-diethyloxacarbocyanine iodide $\left(\mathrm{DiOC}_{2}(3)\right.$ from EMD Millipore, Billerica, MA) were used as fluorescent substrates to assay the MRP1 and MDR1 transport activity, respectively, by flow cytometry. Cells were exposed to solvent or HDAC inhibitor for 48 hrs at $37^{\circ} \mathrm{C}$, harvested and resuspended in fresh RPMI 1640 medium containing either $1 \mu \mathrm{M}$ CFDA or $0.5 \mu \mathrm{g} /$ $\mathrm{ml} \mathrm{DiOC}_{2}(3)$ and kept on ice for 20 min to allow their uptake/influx. Cells were centrifuged, washed with cold PBS and resuspended in $1 \mathrm{ml}$ RPMI 1640 medium. Each cell suspension was divided into two $0.5 \mathrm{ml}$ aliquots; one set was kept on ice and the other set was shifted to $37^{\circ} \mathrm{C}$ for up to $30 \mathrm{~min}(\mathrm{CFDA})$ or $45 \mathrm{~min}\left(\mathrm{DiOC}_{2}(3)\right)$ to allow temperature-dependent substrate efflux. Cells were centrifuged again at $4^{\circ} \mathrm{C}$, resuspended in $0.4 \mathrm{ml}$ PBS containing $0.1 \mu \mathrm{g} / \mathrm{ml}$ propidium iodide (PI), and analyzed by flow cytometry using excitation/emission wavelength of 488/525 nm for CFDA and DiOC2(3), and 488/617 nm for PI. Live and dead cells were differentiated by inclusion of PI, and only live cells were analyzed.

The above procedure was modified when daunorubicin (Selleck Chemicals, Houston, TX) was used as a substrate for the efflux assay. Cells were exposed to solvent or HDAC inhibitor for $48 \mathrm{hrs}$. Daunorubicin 
$(2 \mu \mathrm{M})$ was added to the cell suspension and incubated for $1.5 \mathrm{hrs}$ at $37^{\circ} \mathrm{C}, 5 \% \mathrm{CO}_{2}$ to allow its uptake. Cells were pelleted, washed with PBS (room temperature) and resuspended in warm RPMI 1640 medium. Cells were further incubated at $37^{\circ} \mathrm{C}, 5 \% \mathrm{CO}_{2}$ to allow daunorubicin efflux. Sample aliquots were taken after 0, 1, 2 and 3 hrs. Cells were centrifuged, resuspended in $0.4 \mathrm{ml}$ PBS containing $2 \mu \mathrm{g} / \mathrm{ml}$ 4', 6-diamidino-2-phenylindole (DAPI) and analyzed by flow cytometry using excitation/emission wavelength of 488/575 nm for daunorubicin and 405/461 $\mathrm{nm}$ for DAPI. Live and dead cells were differentiated by inclusion of DAPI and only live cells were analyzed.

To inhibit efflux of daunorubicin, $2 \mathrm{mM}$ sodium orthovanadate (Selleck Chemicals) was added $1 \mathrm{hr}$ after the addition of daunorubicin and incubated for another 30 min. Efflux assay was performed as described above in the presence of $2 \mathrm{mM}$ sodium orthovanadate.

\section{ACKNOWLEDGMENTS}

Part of this research was performed in the Flow Cytometry \& Cellular Imaging Facility, which is supported in part by the National Institutes of Health through M.D. Anderson's Cancer Center Support Grant CA016672. This work was also supported by the Stephen L. and Lavinia Boyd Fund for Leukemia Research, and by funds donated by grateful patients.

\section{CONFLICTS OF INTEREST}

The authors declare no conflicts of interest.

\section{REFERENCES}

1. Andersson BS, Valdez BC, Jones R. Pharmacologic basis for high-dose chemotherapy. In: Forman SJ, Negrin RS, Antin JH, Appelbaum FR (Eds). Thomas' Hematopoietic Cell Transplantation, 5th edn. Wiley-Blackwell, Oxford, UK, 2016. pp 211-222.

2. Valdez BC, Li Y, Murray D, Champlin RE, Andersson BS. The synergistic cytotoxicity of clofarabine, fludarabine and busulfan in AML cells involves ATM pathway activation and chromatin remodeling. Biochem Pharmacol. 2011; 81:222-32.

3. Valdez BC, Nieto Y, Murray D, Li Y, Wang G, Champlin RE, Andersson BS. Epigenetic modifiers enhance the synergistic cytotoxicity of combined nucleoside analogDNA alkylating agents in lymphoma cell lines. Exp Hematol. 2012; 40:800-10.

4. Nieto Y, Valdez BC, Thall PF, Ahmed S, Jones RB, Hosing C, Popat U, Shpall EJ, Qazilbash M, Gulbis A, Anderlini P, Alousi A, Shah N et al. Vorinostat combined with high-dose gemcitabine, busulfan, and melphalan with autologous stem cell transplantation in patients with refractory lymphomas. Biol Blood Marrow Transplant. 2015; 21:1914-20.
5. Teo EC, Valdez BC, Ji J, Li Y, Liu Y, Brammer JE, Hosing C, Nieto Y, Champlin RE, Andersson BS. Synergistic cytotoxicity of busulfan, melphalan, gemcitabine, panobinostat, and bortezomib in lymphoma cells. Leuk Lymphoma. 2016. In press.

6. Köck K, Grube M, Jedlitschky G, Oevermann L, Siegmund W, Ritter CA, Kroemer HK. Expression of adenosine triphosphate-binding cassette (ABC) drug transporters in peripheral blood cells: relevance for physiology and pharmacotherapy. Clin Pharmacokinet. 2007; 46:449-70.

7. Cole SP, Deeley RG. Transport of glutathione and glutathione conjugates by MRP1. Trends Pharmacol Sci. 2006; 27:438-46.

8. Deeley RG, Westlake C, Cole SP. Transmembrane transport of endo- and xenobiotics by mammalian ATP-binding cassette multidrug resistance proteins. Physiol Rev. 2006; 86:849-99.

9. Munoz M, Henderson M, Haber M, Norris M. Role of the MRP1/ABCC1 multidrug transporter protein in cancer. IUBMB Life. 2007; 59:752-7.

10. Robey RW, Zhan Z, Piekarz RL, Kayastha GL, Fojo T, Bates SE. Increased MDR1 expression in normal and malignant peripheral blood mononuclear cells obtained from patients receiving depsipeptide (FR901228, FK228, NSC630176). Clin Cancer Res. 2006; 12:1547-55.

11. Batty N, Malouf GG, Issa JP. Histone deacetylase inhibitors as anti-neoplastic agents. Cancer Lett. 2009; 280:192-200.

12. Dogan AL, Legrand O, Faussat AM, Perrot JY, Marie JP. Evaluation and comparison of MRP1 activity with three fluorescent dyes and three modulators in leukemic cell lines. Leuk Res. 2004; 28:619-22.

13. Gekeler V, Ise W, Sanders KH, Ulrich WR, Beck J. The leukotriene LTD4 receptor antagonist MK571 specifically modulates MRP associated multidrug resistance. Biochem Biophys Res Commun. 1995; 208:345-52.

14. Minderman H, Suvannasankha A, O'Loughlin KL, Scheffer GL, Scheper RJ, Robey RW, Baer MR. Flow cytometric analysis of breast cancer resistance protein expression and function. Cytometry. 2002; 48:59-65.

15. Sharom FJ. ABC multidrug transporters: structure, function and role in chemoresistance. Pharmacogenomics. 2008; 9:105-27.

16. Hobbs AS, Froehlich JP, Albers RW. Inhibition by vanadate of the reactions catalyzed by the $(\mathrm{Na}++\mathrm{K}+)$-stimulated ATPase. A transient state kinetic characterization. J Biol Chem. 1980; 255:5724-7.

17. Sarkadi B, Price EM, Boucher RC, Germann UA, Scarborough GA. Expression of the human multidrug resistance cDNA in insect cells generates a high activity drug-stimulated membrane ATPase. J Biol Chem. 1992; 267:4854-8.

18. Nieto Y, Valdez BC, Thall PF, Jones RB, Wei A, Myers A, Hosing C, Ahmed S, Popat U, Shpall EJ, Qazilbash M, Gulbis A, Anderlini P, et al. Double epigenetic modulation 
of high-dose chemotherapy with azacitidine and vorinostat for patients with refractory or poor-risk relapsed lymphoma. Cancer. 2016. In press.

19. Paumi CM, Ledford BG, Smitherman PK, Townsend AJ, Morrow CS. Role of multidrug resistance protein 1 (MRP1) and glutathione S-transferase A1-1 in alkylating agent resistance. Kinetics of glutathione conjugate formation and efflux govern differential cellular sensitivity to chlorambucil versus melphalan toxicity. J Biol Chem. 2001; 276:7952-6.

20. Eberharter A, Becker PB. Histone acetylation: a switch between repressive and permissive chromatin. Second in review series on chromatin dynamics. EMBO Rep. 2002; 3:224-9.

21. Miller JL, Grant PA. The role of DNA methylation and histone modifications in transcriptional regulation in humans. Subcell Biochem. 2013; 61:289-317.

22. Vaute O, Nicolas E, Vandel L, Trouche D. Functional and physical interaction between the histone methyl transferase Suv39H1 and histone deacetylases. Nucleic Acids Res. 2002; 30:475-81.

23. Barski A, Cuddapah S, Cui K, Roh TY, Schones DE, Wang Z, Wei G, Chepelev I, Zhao K. High-resolution profiling of histone methylations in the human genome. Cell. 2007; 129:823-37.

24. Koch CM, Andrews RM, Flicek P, Dillon SC, Karaöz U, Clelland GK, Wilcox S, Beare DM, Fowler JC, Couttet P,
James KD, Lefebvre GC, Bruce AW et al. The landscape of histone modifications across $1 \%$ of the human genome in five human cell lines. Genome Res. 2007; 17:691-707.

25. Rosenfeld JA, Wang Z, Schones DE, Zhao K, DeSalle R, Zhang MQ. Determination of enriched histone modifications in non-genic portions of the human genome. BMC Genomics. 2009; 10:143.

26. Leonard GD, Fojo $\mathrm{T}$, Bates SE. The role of $\mathrm{ABC}$ transporters in clinical practice. Oncologist. 2003; 8:411-24.

27. Ravid Z, Goldblum N, Zaizov R, Schlesinger M, Kertes T, Minowada J, Verbi W, Greaves M. Establishment and characterization of a new leukaemic T-cell line (Peer) with an unusual phenotype. Int J Cancer. 1980; 25:705-10.

28. Sundstrom C, Nilsson K. Establishment and characterization of a human histiocytic lymphoma cell line (U-937). Int J Cancer. 1976; 17:565-77.

29. Valdez BC, Li Y, Murray D, Corn P, Champlin RE, Andersson BS. 5-Aza-2'-deoxycytidine sensitizes busulfanresistant myeloid leukemia cells by regulating expression of genes involved in cell cycle checkpoint and apoptosis. Leuk Res. 2010; 34:364-72.

30. Valdez BC, Li Y, Murray D, Ji J, Liu Y, Popat U, Champlin $\mathrm{RE}$, Andersson BS. Comparison of the cytotoxicity of cladribine and clofarabine when combined with fludarabine and busulfan in AML cells: Enhancement of cytotoxicity with epigenetic modulators. Exp Hematol. 2015; 43:448-61. 